\title{
Congestion Control using Cross layer and Stochastic Approach in Distributed Networks
}

\author{
Selvarani R \\ Department of Computer science and Engineering \\ Alliance College of Engineering and Design \\ Bangalore, India
}

\author{
Vinodha K \\ Department of Information science and Engineering \\ The Oxford College of Engineering \\ Bangalore, India
}

\begin{abstract}
In recent past, the current Internet architecture has many challenges in supporting the magnificent network traffic. Among the various that affect the quality of communication in the massive architecture the challenge in maintaining congestion free flow of traffic is one of the major concerns. In this paper, we propose a novel technique to address this issue using cross layer paradigm based on stochastic approach with extended markovian model. The cross layer approach will bridge the physical layer, link layer, network layer and transport layer to control congestion. The resource provisioning operation will be carried out over link layer and the mechanism of exploring the congestion using stochastic approach will be implemented over the network layer. The Markov modeling is adopted to identify the best routes amidst of highly congested paths and it is carried out at the transport layer. An analytical research methodology will be adopted to prove that it is feasible to develop a technique that can identify the origination point of congestion and share the same with the entire network. It is found that this approach for congestion control is effective with respect to end to end delay, packet delivery ratio and processing time.
\end{abstract}

Keywords-Distributed Network System; cross layer; congestion; Traffic Flow; Rate Control Metric

\section{INTRODUCTION}

Internet plays a vital role in dissemination of knowledge and servicing seamless and ubiquitous communication in the present era. With the advancements in technologies like cloud computing and optical network, offering high speed data delivery, data storage and retrieval is not an impossible task [1] yet, there is still a problem with the existing internet architecture. A closer look into the existing internet architecture revels that it is packed with various complexities Viz. incompatible in allowing connectivity with heterogeneous networks and its respective protocols, operating with various distributed networks [2][3]. The existing internet design principles can only permit networking with less complexity in its routing and communication process. These principles are not scalable for the requirement of the future internet architecture. The reason behind this is untrusted communication, more customer-oriented user environment, availability of many commercial network operators, datacentric utilities, and the worst part is intermittent connectivity [4]. Another challenging problem is its inclination towards Internet Protocol (IP) paradigm that makes it suitable for static internet users but not for mobile internet users. Therefore, whenever an application meets heterogeneity, it introduces a great deal of challenges for the network-based architecture and at the same time, it also leads to significant problems of resource allocation that can potentially affect the quality of performance. The connection technique of this architecture is characterized by one-to- many and many-to-many connections and also supports smart virtualization process. The significance of user-based participation is quite high with compatibility of multi-hop transmission scheme [5]. Unfortunately, none of the above mentioned schemes are present even to a lesser extent in the existing internet architecture.

The present paper deals with the problems related to congestion control in future internet architecture. Although understanding the user-behavior over traffic and predicting it is an NP(nondeterministic polynomial time) hard problem, there are studies existing in past that has already focused on congestion control mechanism but less evidence of studies have focused towards congestion control in future internet architecture. Essentially, this is built over three components viz. service, architecture and infrastructure. The next problem is interoperability. Given a scenario of multiple and heterogeneous network, it is a challenging task to process the control messages. This phenomenon is definitely a big impediment towards congestion. The next issue is for a given congestion over the dynamic network, it is quite challenging to maintain a balance between identifying the point of congestion and processing heterogeneous control messages. Hence, it can be said that it is quite a difficult task to identify and mitigate the level of congestion in this architecture.

This paper presents a joint algorithm that incorporates cross layered mechanism with stochastic approach and Markov modeling to mitigate the potential issues of congestion in massive distributed system (future internet architecture). Section II reviews the existing literature for congestion control. The motivation and problem identification is discussed in section III. Section IV deal with the proposed study and its significant contribution. The algorithms that are implemented to attain the goals are presented in section V. The results of the proposed study are analyzed in Section VI. The concluding remarks are discussed in section VII.

\section{RELATED WORK}

The existing research in this area is reveled here.

Gholipour et al. [6] have carried out an investigation on congestion problems in sensor network. The working principal of the sensor network operate with distributed algorithm. Here the authors discussed a technique based on cost metric. The 
results were compared with respect to energy and packet. Efthymiopoulos et al. [7] have presented a study on congestion minimization pertaining to real-time streaming. The authors have introduced a technique that can provide traffic management in different domains of the network based on the bandwidth. The system is purely made for the internet-based peer-to-peer traffic. Jose et al. [8] have presented a congestion minimization technique that evaluates the rate of communicating signals in highly distributed manner. The outcome of the study was evaluated with respect to transmission rate and found that it offers better rate control mechanism for minimizing the congestion. Zaki et al. [9] have presented a solution towards mitigating congestion that is witnessed over highly unpredictable mobile networks. The authors tested their finding over the continuous date occurred on $3 \mathrm{G}$ network. The outcome of the study was evaluated with respect to throughput and delay to find that proposed system offers better resiliency for internet-based congestion. Ichrak et al. [10] have also investigated the problems of congestion in TCP-IP(Transmission Control Protocol/Internet Protocol)based connection. Sonmez et al. [11] have presented a technique that focuses congestion identification and reduction owing to multimedia transmission. The study focuses on the congestion control and its effect on the quality of the transmitted multimedia files using fuzzy logic mechanism. The outcome of the study was evaluated with respect to Peak Signal-to-Noise Ratio (PSNR).

Reddy and Krishna [12] has presented cross layer approach in order to mitigate the congestion issues in mesh network using TCP New Reno protocol. They focused on efficient channel capacity optimizing during the massive multimedia transmission and the results were assessed using packet transmission rate and delay. A scheme for controlling the congestion over TCP-IP based network was presented by the authors Carofiglio et al. [13]. They has used the principle of active queue management to control the congestion and found that the technique possessed an effective window size, round trip time, and queue size. Further studies towards distributed system were carried out by Antoniadis et al. [14]. Although they worked on a small network, the principle applied was considered as a guiding factor for large scale distributed network as it focuses on addressing an effective traffic management technique using game theory. Cai et al. [15] have presented a model for controlling congestion in TCP-based communication system. The proposed methodology controls congestion over the wireless network based on node-to-node interactions. Using the case study of adhoc-based network, they have proved that their method offered better congestion control. Under the constraint of the fading channel, Ye et al [16] used probability theory to show that the congestion control model for vehicular network offered improvement in the energy efficiency and data packet transmission over adhocbased networks. Similar kind of work was carried out by Bouassida and Shawky [17] .They presented dynamic scheduling algorithm based on the priority of the messages. The focused on improving data reliability of real-time vehicular network.

Kas et al. [18] have presented a technique for performing scheduling over dynamic channels. The aim of this is to increase the throughput from application viewpoint. A specific level of weight is assigned to each node that is arbitrarily finetuned based on saturation level of the queue. The results were evaluated with respect to end-to-end delay and packet delivery ratio over Constant Bit Rate traffic. Li et al. [19] have investigated congestion control for delay-based network. The authors have compared their work with respect to voice and data traffic and showed that it can control congestion based on the available delay information. Misra et al. [20] has presented a unique technique based on automata theory for managing the congestion over wired network. The author have also applied stochastic-learning based mechanism and cellular automata for managing an effective queue size. The outcomes were assessed using sequence number, queue factor, etc. Uthra et al. [21] have proposed a rate control mechanism for governing the traffic so that efficient throughput can be managed to ensure transmission free from any sorts of collision. The outcome of the simulation-based study is recorded and compared with the existing predictive-based mechanism to control congestion and found that the presented system minimizes the traffic congestion and also enhances the traffic performance.

The following section presents the problem that is identified after reviewing the work that was carried out by researchers in the field of congestion control.

\section{MOTIVATION AND PROBLEM IDENTIFICATION}

The following are the areas to be considered for efficient performance of future Internet. The prevailing research in this area fail to address the following:-

- The network quality parameters like delay, latency and channel capacity are not considered efficiently for congestion control mechanism.

- The current cross layer design allows manipulation of various layer parameters which leads to complication of congestion control and error management. In addition to that it is observed that the complexity of identifying the source of congestion is difficult because of the inefficient handling of randomness of traffic in heterogeneous network.

\section{PROPOSED SYSTEM}

The aim of the proposed system is to develop a novel algorithm that can identify the origin of congestion in distributed network system. Here, the emphasis on network resource allocation for dynamic data flow control is given. As future internet architecture will possess all the possible complexities of existing internet as well as other networking standards (owing to reconfigurable nature), it is essential to address the issues through empirical and analytical modeling. In addition to regular quality parameters our research addresses the issues related to air medium e.g interference and different levels of noise over wireless channel for modeling the traffic. This paper is a continuation of our work where we have offered a packet level congestion by introducing a parameter i.e., Rate Control Metric (RCM)[24] . This metric is designed to offer an efficient control over the highly distributed network. The system is designed with the principle of cross layer paradigm. The randomness in the heterogeneous network is studied 
through stochastic based probability model. This system is viewed as a massive network through graph theory modeling for better analysis of traffic congestion. In order to study and mitigate the traffic congestion in heterogeneous network (Future internet architecture) the performance metric were analyzed through RCM.

In this research we have considered cross layer approach for effective communication between networks. The resource provisioning technique is enhanced through stochastic based approach where, the model based on markovian modeling provides an optimized search for favorable node for routing. This model supports in identifying the best possible node amidst of congested nodes for speedy transfer of data which enhances the throughput of the network.

\section{A. Cross Layer network model}

Identifying the origination point of congestion and determining the control messages for processing the routing process to mitigate congestion requires a robust mechanism. Cross-layered approach is used to overcome this problem by controlling physical layer, data link layer, network layer, and transport layer in the protocol stack of future internet architecture. The cross layer network model plays an important role in arbitrary provisioning of network resources for congestion control. The presented scheme uses a significant routing factor that supports dynamic communication through multiple hops. It also uses a scheme that controls and manages the rate of traffic flow to achieve the fairness in sharing of network resources. A cost efficient provisioning algorithm is designed that models a novel queuing technique for maintaining queue stability. One of the significant focuses of the presented technique is to include the scenarios of noise and interference. This approach helps in processing control messages of multiple layers to adjust the rate of traffic flow during peak hours and also select favorable nodes for communication between two different networks. Hence, the cross-layer scheme offers flexibility to process the control request with less delay and also ensures that it is applicable for distributed network with heterogeneity.

\section{B. Stochastic Approach}

The stochastic approach of the proposed system mainly involves an integrated implementation of resource provisioning, communication and controlling the traffic directions. This approach initializes the discrete networking states followed by selection of highly stabilized links, and apply provisioning. The system uses graph theory to design an algorithm that works over the distributed machines. The significant contribution of this approach is to develop a network model that uses noise and signal power to categorize the quality of the links. It also consider the constraints of first two layers (link, physical) where there is no assured link rate for assigned time instances in distributed networking system. There is also a possibility that the capacity of the route may vary over a period of time that will lead to a significant stochastic problem. In order to solve this issue, we have implemented Rate Control Metric (RCM) [24] that can extract the exact information about the traffic rate thereby giving more information about the capacity of the routes. In order to solve the problems related to computational complexity, we also initialize a hypothetical matrix that stores and extracts the best provisioned values, which acts as alternative for the congestion states of traffic. Hence, there is no significant control overhead due to this. Moreover, the provisional matrix is regularly updated which makes the proposed system independent from any degree of congestion found in a specific transmission area.

\section{Markov Modelling}

The main aim of Markov Modeling is to optimize the stochastic approach used for congestion control. The goal of this module will be to minimize the end-to-end delay in distributed networking system. We apply probability theory along with Markov model to find out alternate routes by exploring non-congested paths for routing. Markov chain is used for mapping the network model that uses queuing theory over the layered design. (The study doesn't emphasize much on queuing mechanism explicitly as there is already robust mechanism specific to routing protocols in distributed network). The system maintains two types of traffics in a matrix i.e. local traffic and global traffic. Local traffic can be accessed at any instance of time and global traffic information can be accessed only when the node has better residual energy. The energy model based on first order radio model or Radio Frequency (RF) circuitry principle [26] will be implemented in the proposed system. The next section discusses the implementation of cross layer algorithm, stochastic approach algorithm and markov modeling.

\section{ALGORITHM IMPLEMENTATION}

\section{A. Algorithm for Cross-Layer Approach}

In this work, we address the mitigation of congestion in the distributed network system through cross layer approach. The algorithm and implementation are as depicted below.

Algorithm:

Input: $\mathrm{n}$ (nodes), $\rho$ (queue)

Output: updation of Link

Start

1. init $n, \rho, / / n$ is the number of nodes $\& \rho$ is the queue size

2. Estimate generated packets pkt on condition

$p k t=\left[\frac{\rho}{J}\right]_{0}^{I}$

3. Define link cost

$C_{s, d}(t)=\arg _{\max }[\Delta \rho]$

4. $\mathrm{L}[\mathrm{t}] \rightarrow\{\mathrm{Ls}, \mathrm{d}[\mathrm{t}]\}$

5. Define enhancement in cross-layer provisioning

$L_{c}(t)=\int_{0}^{L}\left(\rho[x],\left(L_{o}-L_{i}\right)\right) d x$

6. Select $\rightarrow \operatorname{rand}[\mathrm{t}] \epsilon \mathrm{L} \| \operatorname{rand}[\mathrm{t}]=\mathrm{Lc}[\mathrm{t}]$

7. update $\mathrm{L}[\mathrm{t}]$ 
End

The algorithm is formalized by considering the number of nodes $\mathrm{n}$ and initial queue size $\rho$. Fig.1 illustrates the complete process flow of the proposed cross layer based provisioning.

The queue stability of the distributed networking system is defined by the following equation which is used to filter out all the links that have their queue size tending to infinity.

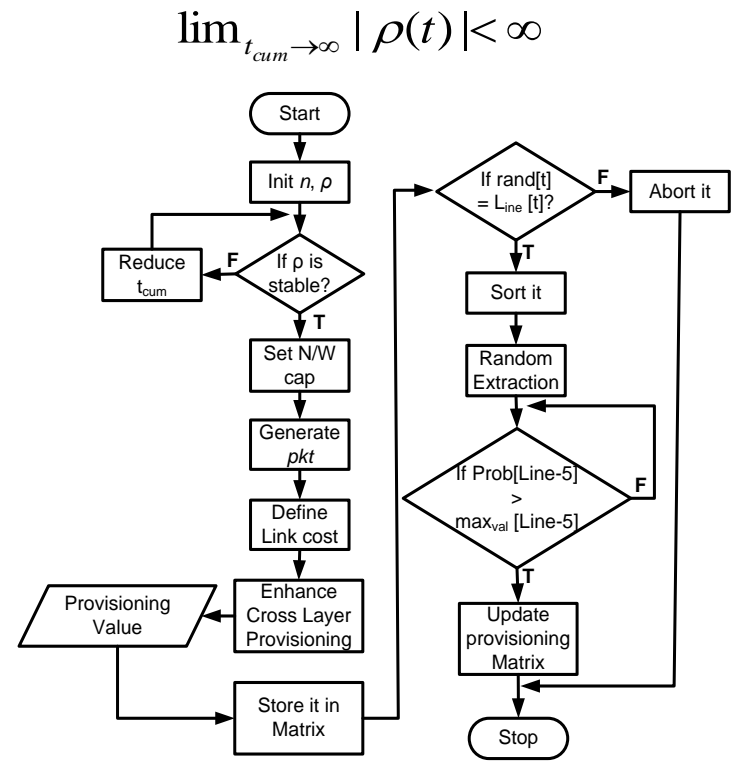

Fig. 1. Process flow for Cross Layer Provisioning

The link capacity of the network is expressed in terms of the pkt. Here, it is assumed that at time t the nodes in the network generate data packets equivalent to queue size $\rho$ with controlled variables $I$ and $J$ ( $\mathrm{I}$ and $\mathrm{J}$ are positive integers) are as shown in line 2 of the algorithm. As the future internet architecture supports higher range of heterogeneity in device integration, there are possibilities of signal collision that leads to channel interference. In order to distinguish the quality of links (or routes) a new measurement link cost $C$ is considered and is estimated based $\Delta \rho$, where the variable $\Delta \rho$ represents difference between the source queue $\rho_{s}$ and destination queue $\rho_{d}$ at the time $t$. The link provider metric $L[t]$ that is equivalent to $L_{\mathrm{s}, \mathrm{d}}[t]$, where $L$ represents a matrix of provider that consist of non-colliding links between any source(s) and destination(d) is considered as the main parameter of the algorithm. It is assumed that initially the buffer is shared among each recipient node. The link provisioning matrix is updated considering the maximum value of the two arguments i.e. queue $\rho[\mathrm{x}]$ and difference between outgoing capacity of link Lo and incoming capacity of link Li.

The link provider will arbitrarily select an element from the matrix that satisfy the condition i.e. probability of selected element is equivalent to enhanced value (Line-6). It is updated as follows

$$
L_{c}(t)=\int_{0}^{L}\left(\rho[x],\left(L_{o}-L_{i}\right)\right) d x
$$

Here, the link metric $\mathrm{L}[\mathrm{t}]$ is estimated in terms of its queue size and the link capacity helps in the route selection process.

\section{B. Algorithm for Stochastic Approach}

In distributed networking system the traffic may undergo uncertainties like dynamic topology, random mobility etc. in high degree of randomness. The state of the network with uncertainty is analyzed through stochastic process in which the future node is identified with the theory of cross layer architecture. The nodes are initialized and their details are maintained on a data structure managed by graph theory. Owing to the distributed nature of the system, we assume that the control messages are free from errors or noise. After the implementation of cross-layer approach, we assume that there is no deviation or variance in the route capacity over the advancement of time.

Algorithm

Input: Es(energy for transmitting), $\delta$ s, d (gain factor of the

power), $\beta$ (capacity of the channel), $\psi$ (noise density)

Output: Provisioning state

Start

1. Evaluate SNR

$S N R_{s, d}=\frac{E_{s} \cdot \delta_{s, d}}{\beta \cdot \psi}$

2. Evaluate capacity of link

$$
\text { Lcap }=\beta \log 2(1+\text { SNRs, })
$$

3. Define duplicated groups

$$
d p=\left\{d p S \mid s, d \in N, d p S \subseteq N_{s} \cap N_{d}\right\}
$$

4. Function for duplicated groups

$$
f(s, d p)=\left\{d p S \mid(s \in d p S)^{\wedge}(d p S \subseteq d p)^{\wedge}(|d p S| \geq 2)\right\}
$$

5. If $s \subseteq S_{\text {Than }}$

6. for all $d i \subseteq D_{\text {do }}$

7. $r c m(t) \leftarrow \operatorname{argmin}(r c m m a x$, scaler_mult $(t))$;

8. Apply Algorithm-1

9. Transmit data from $\mathrm{s}$ to $\mathrm{d}$

10. Update scalar_multi $(t) \rightarrow$ state of provisioning End

The algorithm is implemented by defining a network model, Signal-to-Noise Ratio (SNR) and Link Capacity (Line1 and 2 of the algorithm). The duplicate control messages for analysis purpose are generated using Line-3 of the algorithm. In the above algorithm the source node $s$ is identified as $s_{\mathrm{id}}$ and matrix of duplicate control messages containing information 
about $\mathrm{s}$ as $s . d p$. The duplicated groups are formulated using the equation as shown in Line-4. The cross layer architecture of the future internet is designed in such a way that each source node $\mathrm{s}$ can access its routing table $\mathrm{N}_{\mathrm{s}}$. For reliable routing during peak traffic the algorithm allows node $s$ to construct multiple hops with other nodes for providing alternate routes. The one dimensional matrix is generated by scalar multiplication of $\mathrm{s}$ and $\mathrm{dp}$ and the same is stored at every node. However, for all the duplicate control messages $\mathrm{dpS}$, only the node that has highest value of id is chosen and is used in the computation process. The algorithm looks for all the source nodes $\mathrm{s}$ ( $\mathrm{S}$ is total source nodes) and attempts to control the flow of packets. It then checks all the respective destination nodes and uses rate control metric (RCM) [24] to further enhance the provisioning for the data transmission. Finally, with the help of cross layer provisioning algorithm the date is transmitted towards the destination $\mathrm{d}$.

The significance of the stochastic based approach algorithm is that it further enhances the resource provisioning offered by cross-layer based provisioning technique at the link layer and also supports better communication in the network layer by favoring multiple hops routing in distributed networking system. Finally, the data transmission is improved by applying rate control metric [24] which assigns an appropriate rate at the transport layer for effective end to end communication. Hence, the algorithm completely supports the cross-layer paradigm for future internet architecture to ensure interoperability among heterogeneous networks and achieve efficient data transmission.

\section{Algorithm for Markov Modeling}

The Markov modeling is used to further strengthen the algorithm discussed in the above sections and to apply stochastic modeling to further enhance the congestion control algorithm and offer a better solution to control traffic congestion. In Markov modeling each node is represented as Mc that is composed of the total number of layers corresponding to Lcap+1 (numerically). The amount of data packets pkt processed on each layer should be equivalents to Lcap such that $0<\mathrm{pkt}<\mathrm{Lcap}$. We consider two different forms of layers Viz. passive layer PL and active layer AL. Passive layer represents the passive process when the nodes doesn't have any packet to forward ( $\mathrm{pkt}=0$ ) whereas in active layer, nodes always have packets for forwarding ( $\mathrm{pkt}>0$ ). As the future internet architecture possess different wireless nodes it is assumed that there are other feasible communication outages that will call for retransmission phenomenon. We denote $\varphi$ as the amount of retransmission and $\mathrm{Wn}$ to be amount of unit trail of transmission. The algorithm for Markov modeling is given below.

\section{Algorithm}

Input: pkt (Packet), Lcap (Link capacity w.r.t queue), PL(Passive Layer), AL(Active Layer), $\varphi$ (Maximum amount of retransmission)
Output: Identification of free/busy routes

Start

1. init pkt, Lcap, PL, AL, $\varphi$

2. Determine TP, PP, $\gamma \mathrm{P}, \mathrm{IP}$.

3. Define area of collision A

4. $\{s, d\} \in A_{s, d}, \forall A_{s, d}=A_{s} \cap A_{d}$

5. Obtain $|\mathrm{Fs}, \mathrm{d}|=\mathrm{As} / \mathrm{As}, \mathrm{d}$

6. Evaluate size matrix $|\mathrm{As}, \mathrm{d}|,|\mathrm{Fs}, \mathrm{d}|$, and $|\mathrm{Fd}, \mathrm{s}|$

7. Estimate number of Nodes

size $(|\mathrm{As}, \mathrm{d}|,|\mathrm{Fs}, \mathrm{d}|$, and $|\mathrm{Fd}, \mathrm{s}|)$.network density

8. Estimate the probability of minimum transmission

$p_{A_{s}}=1-\vartheta$

9. Evaluate b1, b2, b3 \& Mc=Algorithm-2 $\{\mathrm{b} 1, \mathrm{~b} 2, \mathrm{~b} 3\}$

10. Find busy routes and free routes.

End

The problem of congestion in future internet architecture leads to network jamming that disrupt the process of identifying the best nodes for forwarding the data packets. This problem can be addressed by designing an algorithm that applies Markov modeling for evaluating the free and busy routes at the peak traffic situation. The algorithm takes the required inputs and computes maximum probability of passive state transition TP, preliminary state component PP, passive state probability component per states $\gamma \mathrm{P}$, and inter-arrival probability IP. Fig. 2 shows the process flow for Markov modeling.

The Markov model is designed by considering three probability matrices viz. b1, b2 and b3. The matrix b1 and b2 represents the probability of a node identifying the busy channel in the first and second Markov process. The matrix b3 represents the feasibility that the packet forwarding process fails due to data packet collision or interference or noise. Line3 shows the collision area A for the source node s. The area A is defined as a transmission zone where there is interference of the neighboring nodes resulting in traffic congestion in that particular transmission area. Hence, area A represents the possible congestion area. As shown in Line-4, it can be interpreted that both the sender node $\mathrm{s}$ and destination node $\mathrm{d}$ will lie within As,d. There can also be another possible transmission zone Fs, d as per Line-5 which may be undetected in the area As.d. It means that there may be an area e.g. F, which goes undetected and the state of congestion is not determined owing to dynamic mobility of nodes in mobile networks. In this case a source node or any intermediate node in area $F_{s, d}$ cannot forward the message to destination node $d$. 


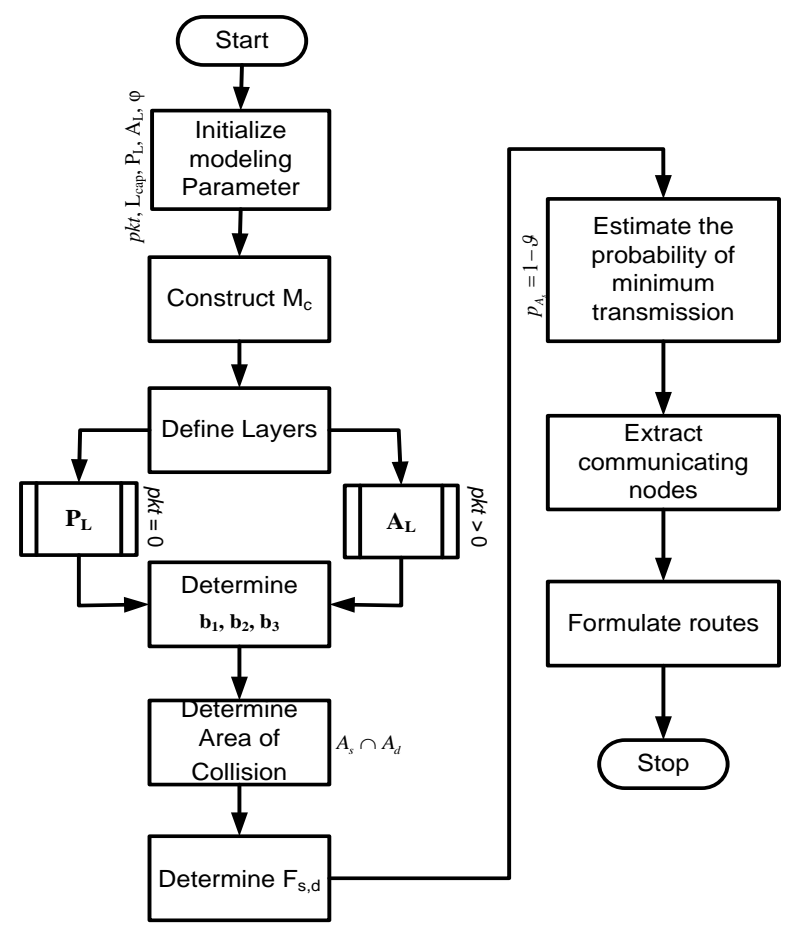

Fig. 2. Process Flow for Markov Modeling

The next phase of the algorithm is to compute the size of the transmission zones as per line- 6 . The algorithm computes the number of nodes in transmission zones by scalar multiplication of network size and network density as per line7. The probability of minimum number of nodes required for forwarding data packets is computed as per Line- 8 . We use a simple variable $\vartheta$ that is equivalent to summation of the probability of all nodes carrying out data packet forwarding divided by total probability of the nodes forwarding data packets from the congested area. This phenomenon will mean that proposed Markov modeling attempts to find the existence of atleast one node which is in fair position to perform data transmission. The Markov modeling proceeds further to find similar kind of nodes and updates the matrix of data communication path that was previously managed by the algorithm of stochastic approach. The updated matrices helps to find the links between favorable nodes as the best possible alternate routes for packet forwarding during the peak traffic condition.

\section{RESULTS AND DISCUSSION}

This section discusses about the results generated from the network simulation through NS2 simulator. The simulation parameters are as shown in Table 1.
TABLE. I. SIMULATION PARAMETERS

\begin{tabular}{|c|c|c|c|}
\hline Parameter & Value & Parameter & Value \\
\hline $\begin{array}{l}\text { Network area( } \\
\text { Simulation) area }\end{array}$ & $\begin{array}{l}1000 \mathrm{x} \\
1200 \mathrm{~m} 2\end{array}$ & $\begin{array}{l}\text { Control packet } \\
\text { size }\end{array}$ & 32 bits \\
\hline Simulation Time & $\begin{array}{r}200 \\
\text { seconds }\end{array}$ & Data packet size & $\begin{array}{l}2000 \\
\text { bytes }\end{array}$ \\
\hline Routing Protocol & NetFlow & Antenna Model & $\begin{array}{r}\text { Omni- } \\
\text { directional }\end{array}$ \\
\hline Pathloss exponent & 0.5 & $\begin{array}{l}\text { Maximum Speed } \\
\text { of node }\end{array}$ & $50 \mathrm{~m} / \mathrm{s}$ \\
\hline MAC Type & 802.11 & $\begin{array}{l}\text { Minimum Speed } \\
\text { node }\end{array}$ & $1 \mathrm{~m} / \mathrm{s}$ \\
\hline Traffic Model & CBR/VBR & $\begin{array}{l}\text { Transmission } \\
\text { range }\end{array}$ & $10 \mathrm{~m}$ \\
\hline Mobility Model & Random & $\begin{array}{l}\text { Transmission } \\
\text { Energy } \\
\text { consumption }\end{array}$ & $0.5 \mathrm{~J}$ \\
\hline Channel Model & Urban & $\begin{array}{l}\text { Receiving Energy } \\
\text { consumption }\end{array}$ & $0.25 \mathrm{~J}$ \\
\hline Channel capacity & $300 \mathrm{Mbps}$ & $\begin{array}{l}\text { Ideal mode } \\
\text { Energy } \\
\text { consumption }\end{array}$ & $0.035 \mathrm{~J}$ \\
\hline \multirow[t]{2}{*}{$\begin{array}{l}\text { Channel sensing } \\
\text { time }\end{array}$} & $0.2 \mathrm{sec}$ & $\begin{array}{l}\text { Sleep mode } \\
\text { Power } \\
\text { consumption }\end{array}$ & $0.02 \mathrm{~J}$ \\
\hline & & $\begin{array}{l}\text { Initial battery } \\
\text { Energy of each } \\
\text { node }\end{array}$ & $10 \mathrm{~J}$ \\
\hline
\end{tabular}

The proposed work focuses in finding an effective solution for congestion control in distributed networking system. The performance parameters like packet delivery ratio, end-to-end delay and processing time are considered to analyze the effectiveness of the proposed system. It is benchmarked with similar studies of Otoshi et al. [25] and Sahuquillo et al. [27]. Otoshi et al. [25] who have presented a stochastic modeling with predictive analysis for identifying discrete states of traffic in distributed networking system. This technique has used a predictive control scheme to minimize the possibilities of predictive error considering network constraints e.g number of hops, length of the hops etc. The mean length of the hops was considered as cost function, which was subjected to optimization using CPLEX solver. The outcome of the work was quite convincing as it has offered better scalability for future internet architecture. Similarly, we consider the work carried out by Sahuquillo et al. [27] as it offers solution to the congestion control for a practical case study of distributed networking system eg. High Performance Computing. The authors have used a mechanism that integrates injection throttle and segregation of congested traffic. We perform a minor modification to techniques introduced in [25] [27] in order to make a suitable testbed for carrying out the comparative analysis. The parameters considered for analysis are end-to-end delay, packet delivery ratio and processing time. 


\section{A. Comparative Analysis of End-to-End Delay}

The end to end analysis is carried out by transmitting the test data of 2000 bytes. The result is as shown in Fig.3.

The graph shows that proposed system is able to minimize the end-to-end delay to a larger extent as compared to existing studies of Otoshi et al. [25] and Sahuquillo et al. [27]. The reason behind this is the technique that is adopted for processing search request and control messages by the proposed system.

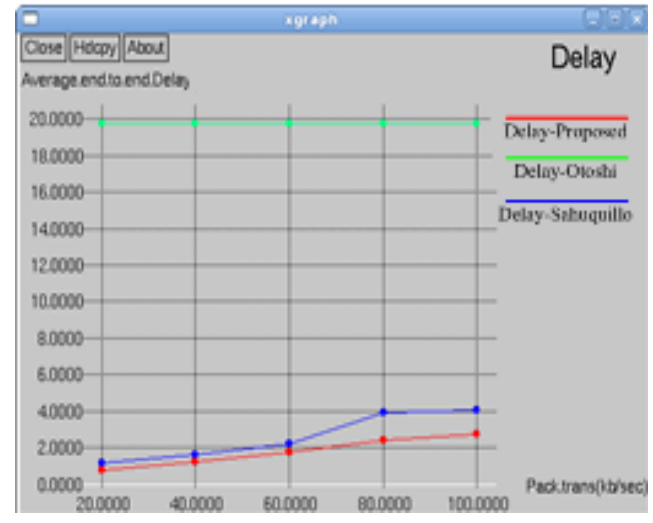

Fig. 3. Comparative Analysis of Delay (sec)

In the proposed system, owing to Markov modeling, it becomes essential for a node to obtain the significant address information of another communication node which could possible reside in transmission zone of $\mathrm{F}_{\mathrm{s}, \mathrm{d}}$ or $\mathrm{A}_{\mathrm{s}, \mathrm{d}}$. As both $\mathrm{F}_{\mathrm{s}, \mathrm{d}}$ and $\mathrm{A}_{\mathrm{s}, \mathrm{d}}$ are different transmission zones, extraction of the node address will be a quite difficult. We simplify this problem by developing a cross layer paradigm that can carry out the task of processing control messages in transport layer thereby minimizing the complexity.

Here, the task of one layer is to aggregate the respective addresses of the nodes and keep on exchanging it with other layers. This operation of interoperability is managed by the network layer. It is the responsibility of the network layer for carrying out the processing of control message as it maintains the communication standards of each transmission zone. This process helps in identifying the point of congestion and makes it aware to the entire network. This process has two advantages viz. i) all nodes can quickly decide about alternate routes and decrease the impact of congestion during peak traffic and ii) degree of congestion at the origination point is reduced by implementing active queue management that directs the packets from highly congested area to less congested point. Hence, end-to-end delay of the proposed work is reduced in the presence of mobility of the nodes which varies at every simulation track points. The problem explored in Otoshi et al. [25] is a predictive scheme. Here, the stochastic processing is adapted to predict and identify the possible prediction error. Hence, the delay factor using this technique cannot be implemented for distributed system of dynamic nature like that of future internet architecture. Similarly, the work done by Sahuquillo et al. [27] have focused on identifying congestion by using control messages which is quite time consuming in its nature. Using Markov modeling, proposed system offers optimized solution for identifying the point of congested and also offers best quality routes for packet forwarding thereby reducing the delay.

\section{B. Comparative Analysis of Packet Delivery Ratio}

Packet delivery ratio is computed by analyzing the amount of data packets received by the destination node to total amount of data transmitted by the source node. The result shown in Fig.4 exhibits that the proposed system offers better packet delivery ratio compared to Otoshi et al. [25] and Sahuquillo et al. [27]. This is because the proposed system provides a better processing of data generated by multiple networking domains in future internet architecture through cross layer paradigm. We start by analyzing the work done by Sahuquillo et al. [27]. The authors have implemented a technique where the incoming packets are organized at the input ports of the switches. The system emphasizes more on organization and less on queuing. This operation when implemented in our scenario reduces the packet delivery ratio. Moreover, the process of identification of the congestion and notify it to other nodes for updates are not discussed in that paper [27]. It is also not sure whether the updates were done over the highly congested area. This issue creates a negative impact on other neighboring nodes by consuming more time to take decision for routing. Hence, packet delivery ratio will be affected when this technique is used in future internet architecture.

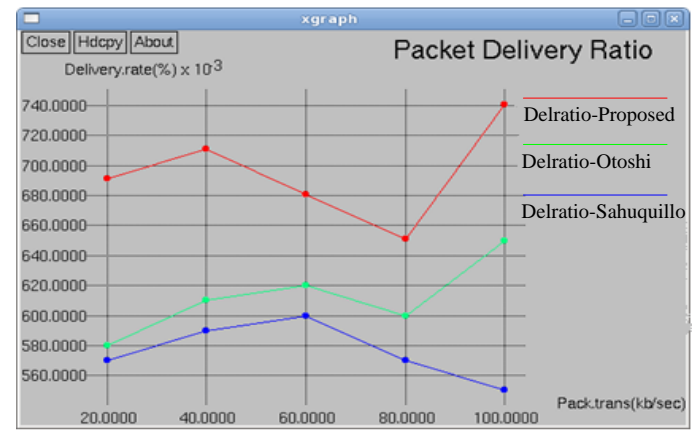

Fig. 4. Analysis of Packet Delivery Ratio

The technique proposed by Otoshi et al. [25] has used the concept of traffic engineering. This technique was implemented through stochastic modeling which is more predictive in nature. The predictive analytic model is assessed for its accuracy of traffic modeling using randomness by adopting traffic engineering with cost as a function on the stochastic model. This is much better than the technique discussed by Sahuquillo et al. [27] as it can accomplish better packet delivery ratio. The main drawback of this technique is that it uses control server to optimize the cost function which leads to less efficient distributed routing. Although, the authors have used relaxation mechanism to sort out this problem, but the probability factors assumed is less when compared to realtime traffic constraints. Hence, its packet delivery ratio is not better than the proposed system. The proposed system overcomes this problem by the algorithm-2 (stochastic) and algorithm-3 (Markov Modeling). These algorithms assist in identifying the best possible routes from non-congested area as well as congested area. The updating mechanism is quite instantaneous with a pause time of 0.0025 seconds in 
simulation study that leads to better packet delivery ratio for a longer period of time.

\section{Analysis of Processing Time}

It is known that an effective congestion control mechanism must have a reduced processing time as far as possible. Lower the processing time means the network can ensure better instantaneous data delivery process. We analyze the processing time with increasing traffic load (packets per seconds). A closer look into the Fig 5 shows that processing time gets reduced linearly with increasing traffic load, which is one of the unique patterns of the proposed study. Usually with increased network traffic, the processing time should be increasing but due to cross layer approach the time complexity is reduced.

The cross layer approach bridges physical layer, link layer, network layer and transport layer. The provisioning operation is carried out over link layer, the mechanism of exploring the congestion using stochastic is implemented over network layer and Markov modeling for further optimizing the best routes (even from highly congested area) is carried out at the transport layer.

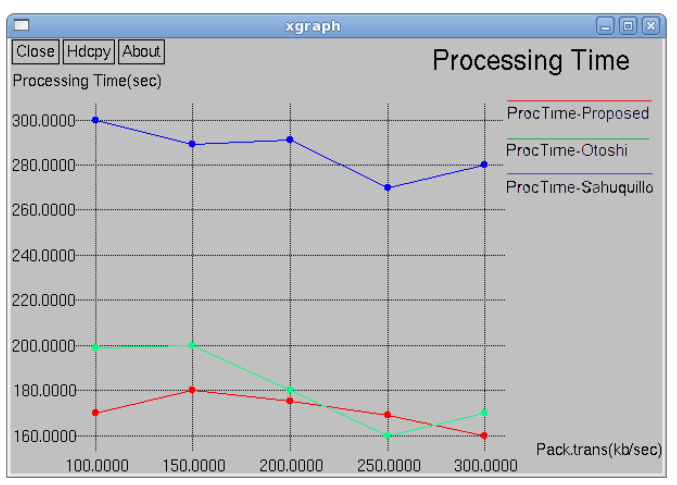

Fig. 5. Analysis of Processing Time

Hence, the system maintains different functionalities over different layers of protocol stack resulting in reduced processing time in the proposed system. For a given simulation environment, Otoshi et al. [25] and Sahuquillo et al. [27] work doesn't meets the demands of the distributed traffic scenario with dense congestion leading to higher processing time when compared to proposed system.

\section{CONCLUSION}

Owing to the complexity in the design principle of distributed networking systems e.g. future internet architecture, the existing algorithms and techniques do not provide solution for mitigating congestion. The proposed system, therefore, presents a technique that uses conglomeration of cross layered approach, stochastic approach, and Markov modeling for addressing the problems of congestion in highly distributed networking system. We have adopted an analytical research methodology to prove that it is feasible to develop a technique that can identify the origination point of congestion and share the same with the entire network. The interesting point of implementation is that proposed technique attempts to use the existing network resources for harnessing the channel capacity in accordance with the state identified by the proposed system.
The outcome of the study were compared with existing system respect to end-to-end delay, packet delivery ratio, and processing time and found that proposed system offers better solution for congestion control.

\section{REFERENCES}

[1] C. White, Data Communications and Computer Networks: A Business User's Approach, Cengage Learning, Computers, 2015

[2] P. Verissimo, L. Rodrigues, Distributed Systems for System Architects, Springer Science \& Business Media, Computers, 2012

[3] N. Rajan, The Digitized Imagination: Encounters with the Virtual World, Taylor \& Francis, Social Science, 2012

[4] J. Holler, V. Tsiatsis, C. Mulligan, S. Avesand, S. Karnouskos, D. Boyle, "From Machine-to-Machine to the Internet of Things: Introduction to a New Age of Intelligence", Academic Press, Technology \& Engineering, 2014

[5] H-Y Wei, J. Rykowski, S. Dixit, WiFi, WiMAX and LTE Multi-hop Mesh Networks: Basic Communication Protocols and Application Areas, John Wiley \& Sons, Technology \& Engineering, 2013

[6] M. Gholipour, A. T. Haghighat, M. R. Meybodi, "Hop-by-hop trafficaware routing to congestion control in wireless sensor networks", Springer- Eurasip Journal on Wireless Communications and Networking, vol.15, 2015

[7] N. Efthymiopoulos, A. Christakidis, M. Efthymiopoulou, "Congestion Control For P2P Live Streaming", International Journal of Peer to Peer Networksm, Vol.6, No.2, August 2015

[8] L. Jose, L. Yan, M. Alizadehy, G. Varghese, "High Speed Networks Need Proactive Congestion Control", ACM-Proceedings of $14^{\text {th }}$ ACM Workshop on Hot Topics in Network Article, No.4, 2015

[9] Y. Zaki, T. Potsch, J. Chen, "Adaptive Congestion Control for Unpredictable Cellular Networks", ACM-SIGCOMM, 2015

[10] T. Ichrak, S. Nawal and A. Mustapha, "Systematic Mapping Study on the Congestion Control Problem in TCP/IP", Contemporary Engineering Sciences, Vol. 7, no. 27, pp.1509-1515, 2014

[11] C. Sonmez, O. D. Incel, S. Isik, M. Y. Donmez, "Fuzzy-based congestion control for wireless multimedia sensor networks", SpringerEurasip Journal on Wireless Communications and Networkin, vol. 63, 2014

[12] C. P. Reddy, P. V. Krishna, "Cross Layer Based Congestion Control in Wireless Mesh Networks", Cybernetics And Information Technologies, Vol.14, No 2, 2014

[13] G. Carofiglio, M. Gallox, L. Muscariellox and M. Papaliniz, "Multipath Congestion Control in Content-Centric Networks", IEEE-Conference on Computer Communication Workshop, pp.363-368, 2013

[14] P. Antoniadis, S. Fdida, C. Griffin, Y. Jin, "Distributed medium access control with conditionally altruistic users", Springer- Eurasip Journal onWireless Communications and Networking, vol.202, 2013

[15] Y. Cai, S. Jiang, Q. Guan, and F R. Yu, "Decoupling congestion control from TCP (semi-TCP) for multi-hop wireless networks", SpringerEurasip Journal on Wireless Communications and Networking, vol. 149, 2013

[16] F. Ye, R. Yim, J. Zhang, S. Roy, "Congestion Control to Achieve Optimal Broadcast Efficiency in VANETs", IEEE-International Conference on Communication, pp.1-5, 2010

[17] M. S. Bouassida and M. Shawky, "A Cooperative Congestion Control Approach within VANETs: Formal Verification and Performance Evaluation", Hindawi Publishing Corporation, Eurasip Journal on Wireless Communications and Networking, Article ID 712525, 2010

[18] M. Kas, I. Korpeoglu, and E. Karasan, "Utilization-Based Dynamic Scheduling Algorithm for WirelessMesh Networks", Hindawi Publishing Corporation, Eurasip Journal on Wireless Communications and Networking, Article ID 312828, 2010

[19] Y. Li, A. Papachristodoulou, M. Chiang, A. R. Calderbank, "Congestion control and its stability in networks with delay sensitive traffic", Elsevier-Computer Networks, vol.55, pp.20-32, 2011

[20] S. Misra, B. J. Oommen, S. Yanamandra, "Random Early Detection for Congestion Avoidance in Wired Networks: A Discretized Pursuit 
Learning-Automata-Like Solution", IEEE Transactions On Systems, Man, And Cybernetics, vol. 40, no. 1, February 2010

[21] R. A. Uthra, S. V. K. Raja, A. Jeyasekar, A. J. Lattanze, "A probabilistic approach for predictive congestion control in wireless sensor networks", Journal of Zhejiang University-Science, vol.15, Iss.3, pp.:187-199, 2014

[22] K.Vinodha, R. Selvarani, "Congestion Control in Distributed Networksa comparative study", Springer-Advances in Computer Science and Information Technology, pp.115-123, 2012

[23] K.Vinodha, R. Selvarani, "Congestion Control in Distributed Networking System-A Review", International Journal of Computer Applications, Vol.83, No 6, December 2013
[24] S. Rangaswamy and V. Krishnareddy, "An efficient traffic regulation mechanism for distributed networks", Springer- Eurasip Journal on Wireless Communications and Networking, vol.154, 2015

[25] T. Otoshiy, Y. Ohsitay, M. Muratay, "Traffic Engineering Based on Stochastic Model Predictive Control for Uncertain Traffic Change" IEEE International Symposium on Integrated Network Management, pp.1165-1170, 2015

[26] A. Grebennikov, N. Kumar, B. S. Yarman, Broadband RF and Microwave Amplifiers, Taylor \& Francis, Computers, 2015

[27] J. E.Sahuquillo, E. G. Gran, P. J. Garcia, "Efficient and Cost-Effective Hybrid Congestion Control for HPC Interconnection Networks", IEEE Transactions On Parallel And Distributed Systems, vol. 26, no. 1, January 2015 